\title{
Measuring the skin microbiome after a $3 \%$ aqueous hydrogen peroxide (H202) dysbiosis using ATP biofluorescence
}

James V Gruber ( $\sim$ vgruber@botanicalsplus.com )

BotanicalsPlus

Jed Riemer

Jeen International

\section{Research Article}

Keywords: Skin Microbiome, ATP Biofluorescence, Rapid Measuring, Dysbiosis

Posted Date: February 10th, 2022

DOI: https://doi.org/10.21203/rs.3.rs-1325039/v1

License: (c) (i) This work is licensed under a Creative Commons Attribution 4.0 International License.

Read Full License 


\section{Abstract}

Background: Since its complete mapping in 2008, the human skin microbiome has become an important area of research related to skin health. The human skin is populated by an environment of microorganisms, fungi, insects, and viruses that is collectively known as the microbiome. Measuring the skin's microbial diversity can be done but is a sophisticated technique that is performed using expensive instruments that can detect the $16 \mathrm{~S}$ ribosomal RNA of the microorganisms. Finding more rapid and less costly methods to analyze the changes in the skin's microbial biome is desirable.

Results: A study was conducted on thirty (30) inner volar forearms to determine whether Adenosine Triphospate (ATP) biofluorescence could be employed to examine skin microbial dysbiosis caused by the application of $3 \%$ hydrogen peroxide. Fifteen individuals were examined on both arms, both arms having a test site and a control site, for a total of thirty inner volar forearms using a Charm Science NovaLum ATP analyzer [Andover, MA] to examine in a broad sense the skin's total microbial population and how it is affected after surface treatment with $3 \%$ hydrogen peroxide over a 24 -hour period. It was found that surface treatment of the skin with three cotton swab applications of $3 \%$ hydrogen peroxide five minutes apart was able to statistically significantly suppress the expression of ATP fluorescence compared against non-swabbed sites, and the effects remained significant for six hours following the $\mathrm{H}_{2} \mathrm{O}_{2}$ treatment. After 8 hours and 24 hours, the ATP biofluorescence difference returned to nonstatistical significance, indicating the potential return of the stable microbiome.

Conclusion: Using ATP biofluorescence to detect possible sanitizer-induced microbial dysbiosis may be a rapid way to examine how skin treatments may impact the return of microbially disrupted skin to its normal state and how surface treatments may impact the rate of return to normal after a disruptive event.

\section{Background}

Since the groundbreaking studies in 2008 by Elizabeth Grice at the University of Pennsylvania to map the total human skin microbiome, interest in the relationship between the microbiome and skin health has exploded [1,2]. Grice's studies clearly demonstrated that different areas of the body carry different populations of microorganisms depending on several factors, such as body moisture, oil content and exposure to ultraviolet radiation. Interest in the role of the skin's microbiome and how it relates to the skin's health are very important and continue to expand. For instance, it is now well established that skin suffering from atopic dermatitis will typically have an abnormally high population of Staphylococcus aureus [3]. Likewise, there appears to be a link between acne and the occurrence of Cutibacterium acnes (formerly Propionibacterium acnes) [4,5]. Dandruff has been linked to the presence of Malassesia fungi, which are known to be present in higher numbers in populations of people with the condition $[6,7]$. However, whether these opportunistic microorganisms lead to skin dysfunction, or the occurrence of abnormal microorganism populations is due to problems with the skin's innate health remains elusive. 
The skin microbiome is populated by over 19 phyla of microorganisms, with the four dominant ones Actinobacteria, Bacteroidetes, Firmicutes, and Proteobacteria typically comprising over $95 \%$ of the phyla present on healthy skin $[1,5]$. Measuring the diversity of microorganisms on the skin is tricky and requires careful swabbing techniques followed by analysis of the various microorganism populations using $16 \mathrm{~S}$ ribosomal RNA sequencing $[8,9]$. These unique instruments are expensive and require time and skill to use. Other methods to examine more global populations of skin microbes can include agar plating or even the application of newer microbial testing strips, such as the $3 \mathrm{M}$ Petrifilm ${ }^{\grave{O}}$, which allow for more rapid examination of microbial contamination in a global fashion [10]. Even these unique testing techniques require that the skin be carefully swabbed, and the swab is applied to the strips, which must then be cultured over a 24-hour period to determine the microbial content. Importantly, some skin commensal microorganisms do not grow readily outside of the milieu of the skin. Therefore, the use of plating techniques presents some of its own problems. Additionally, while these strips are useful for determining if something is contaminated, quantitatively determining the level of microbial contamination requires counting the colony forming units (CFU) that grow on the strips.

The development of methods to detect microbial ATP has expanded, and there are now several instruments that are offered commercially that use ATP biofluorescence as a means of detecting microbial contamination on surfaces. For example, $3 \mathrm{M}$ offers their Clean-Trace ${ }^{\mathrm{O}}$ Surface ATP Test, and Charm Science ${ }^{\mathrm{O}}$ offers the PocketSwab ${ }^{\mathrm{O}}$ Plus and NovaLum ${ }^{\grave{O}}$ ATP analyzer for use principally in production plants requiring standardized and monitored sanitizing operations. The units are commonly used in many cosmetic and food manufacturing companies to monitor equipment and laboratory surface sanitizing operations. These units offer a very rapid way of swabbing surfaces and then analyzing the overall microbial content on the surface. Studies that have examined the relationship between the relative fluorescence units (RFU) expressed by these ATP instruments and the overall colony forming units (CFU) have shown that the instruments have good sensitivity and linearity between the two measurements $[11,12]$. Use of these instruments to examine oral cavity microbial burden after mouth rinsing, cow teats for microbial cleanliness and to monitor the cleanliness of poultry carcasses in poultry processing suggested that they might have potential for examination of the human skin microbiome [13-15]. This paper will discuss a pilot study intended to examine the possibility of examining skin microbial dysbiosis by disruption of the skin's microbiome with a well-known sanitizing agent, $3 \%$ hydrogen peroxide, and monitoring how quickly the disrupted skin is able to return to normal after the disruption.

\section{Materials And Methods}

\section{Participant Selection}

This study employed the use of fifteen individuals ( 9 males, 6 females, ages 21-61 years, Fitzpatrick Skin Types II-III years) who all signed informed consent to participate in the study. All the participants had healthy, undisrupted inner volar forearm skin and had not taken antibiotics or used any type of potential sanitizing or exfoliating products two weeks prior to participation in the study. Both arms of each participant were used that allowed inclusion of both a test site and a control, untreated, site that allowed 
for a total of $n=30$ inner volar forearms to be examined, as shown in Figure 4. Prior to participating in the study, everyone was instructed to do their routine morning washing routine but to not use soap or scrub on their inner volar forearms to rinse the arms with warm water and to not apply any types of products to their arms for the duration of the study.

\section{Charm Science PocketSwabs and NovaLum ATP Analyzer}

For these studies, a NovaLum VX-II ATP Analyzer from Charm Sciences, Inc. [Andover, MA] was employed to measure ATP biofluorescence. The company also provides PocketSwabÒ Plus swabs. A picture of a PocketSwab is shown below in Figure 5.

PocketSwab Plus is a self-contained unit that has a sterile swab stored until use in a sterile chamber. When ready for use, the swab is withdrawn from the unit and is applied to the surface being tested using specific directions offered by the company, which includes a swabbing pattern that requires approximately sixteen square centimeters of surface while rotating the swab and applying a specific amount of pressure to the surface. The resulting contaminated swab is then inserted back into the unit, and with a twisting screw, motion is thrust through a thin foil membrane that houses an oxidizing fluid. The tip of the swab and the oxidizing fluid drop into the clear chamber at the bottom of the device. The device houses a small tablet that contains a luciferin dye that is activated by the oxidizing fluid and a luciferase enzyme pellet that has been designed to detect adenosine triphosphate (ATP). The device then fluoresces in accordance with the amount of ATP present on the swab. The resulting activated unit is placed into the NovaLum ATP analyzer shown in Figure 6. The device then measures the amount of luciferin biofluorescence and reports the values in relative fluorescent units (RFUs), which can be recorded for testing purposes.

All measurements were made at a room temperature of $22-24{ }^{\circ} \mathrm{C}$ and an $\mathrm{RH}$ of approximately $45-50 \%$, and each participant equilibrated to the room for 15 minutes prior to measurements being taken. The participants were tested initially at all four inner volar forearm locations before any treatments to obtain a baseline level of ATP based on the RFUs noted from the unit for each site. Each participant was then swabbed with a cotton ball saturated with $3 \%$ hydrogen peroxide at the two test sites noted in Figure 1. The adjacent site was not swabbed and remained as an untreated control site on each arm. The participants waited five minutes, and the two sites were swabbed with hydrogen peroxide again. This sanitizing treatment was repeated a third time, and then the participants could continue their normal activities for one hour with the stipulation that they take care not to rub their arms against any solid surfaces or any other skin surfaces for the duration of the first day of the study. The participants offered both arms, which doubled the number of treatment sites, so a total of 30 arms were tested. The two arm sites were also alternated, as shown in Figure 1. Each participant was then measured using the Novalum ATP Analyzer with a single swab at each site at the following timepoints: T(1): $1 \mathrm{hr}, \mathrm{T}(2): 3 \mathrm{hrs}, \mathrm{T}(3): 6 \mathrm{hrs}$, $\mathrm{T}(4)$ : 8 hrs and $\mathrm{T}(5): 24 \mathrm{hrs}$. 
The RFUs for each timepoint were collected and averaged. A total of twelve total measurements $(T(0)$ $\mathrm{T}(5)$ ) were made through the course of each individual study using both arms, and the results for each time point were averaged. The results were then compared between the test sites $\left(\mathrm{H}_{2} \mathrm{O}_{2}\right.$-treated $)$ and the control sites. In addition, the results at Timepoints $T(1), T(2), T(3), T(4)$ and $T(5)$ were normalized by dividing the average RFUs for each timepoint measurement by the averaged baseline measurement to provide normalized hourly comparisons accounting for initial variations in site ATP levels. This provided a unitless measure of normalized data for each timepoint. Using the statistical function in Microsoft Excel, the Student Paired, two-tailed T-Test was employed to determine $95 \%$ confidence $(p \leq 0.05)$ for each data point.

\section{Results}

Figure 1 shows the raw values for the averages of all measurements without normalization of the various timepoints against the initial baseline measurements for each site. The data indicate that only

The Baseline versus Test 1-Hour and Baseline versus Test 3-Hour measurements (green p-values) are significantly different from one another. This indicates that the $3 \%$ hydrogen peroxide treatment reduces the level of ATP fluorescence compared to the control sites that were not treated with peroxide. The data also indicate that the slight increase in average RFU values seen at times $\mathrm{T}(3)$ and $\mathrm{T}(4)$, which are six hours and eight hours post-peroxide treatment, respectively, while appearing slightly elevated, are not statistically significant from the original baseline measurements.

The results of the baseline-normalized ATP RFU measurements post-hydrogen peroxide treatment for the various time points, $T(1)-T(5)$, are shown in Figure 2 . These data more clearly show the indication that the 1-hour and 3-hour peroxide-treated sites (green p-values) show an overall suppression of ATP bioluminescence compared with the control (non-peroxide treated) sites.

Examining the average relative fluorescence units (RFU) of the initial baseline measurements versus similar measurements 24 hours later demonstrated that within all measurable parameters, the initial measurements were identical to the testing results taken 24 hours after the skin had been treated with $3 \%$ hydrogen peroxide (Figure 3).

\section{Discussion}

There is a very important potential source of error that can occur in using ATP biofluorescence to detect human skin microbes: human skin cells also make ATP. It is very likely that ATP coming from the epidermis can find its way to the surface of the skin's stratum corneum and thus complicate measuring microbial ATP versus what is known as somatic skin cell ATP [16]. This problem became acute when $70 \%$ aqueous 2-propanol was employed to try and sanitize the skin's surface. Skin sanitized with $70 \%$ aqueous 2-propanol provided very unusual and conflicting testing results (data not shown). After discussing the testing methods more deeply, it was felt that to employ this technique, the method of sanitization of the 
skin must kill the surface microbes while not significantly disrupting the skin's lipid bilayer. One possible solution was to consider using mild surfactants. However, work published in 2016 by Two et al. demonstrated that mild surfactants did not seem to impact the skin microbiome when measured using the expression of LL37, an antimicrobial peptide expressed in the skin [17]. In the conclusion of these surfactant studies, it was suggested that the "deeper bacteria" in the skin might be able to "rapidly repopulate the surface" of the skin. However, in this interesting microbiome work, the timeframe for this repopulation was not discussed.

In an interesting study published in 2017 through Becker's Hospital Review, Liceaga et al. examined the effectiveness of various aqueous sanitizing ingredients, including antiseptic sanitizers containing chlorhexidine and ozonized water, both in the presence and absence of soap and using simple tap water with soap alone as a control [18]. The authors employed an ATP analyzer [Hygiena's SystemSURE plus ATP Cleaning Verification System] that examined the levels of ATP on the hands after the treatments. The studies employed ten people. The authors of this study noted the following in their study review: "The skin as a living organ has naturally occurring levels of ATP that should not [be] attributed to exogenous microorganisms, eg. virus, fungi, spores, or bacteria. It is believed impossible to eliminate all ATP from skin cells to achieve a zero "0" RLU result". They recognized that the skin produces natural ATP but felt that the impact of the somatic ATP was likely small. The study focused principally on the cleansing properties of the cleansers and not on how the microbiome might respond to the treatments.

It was then felt that perhaps treatment of the skin with a commonly used sanitizing spray containing $3 \%$ hydrogen peroxide might be able to effectively remove surface microorganisms while maintaining the viable pool of deeper microorganisms that would repopulate the skin. An initial study using the product as a spray demonstrated that it was difficult to control the spray, so it was decided that application of $3 \%$ hydrogen peroxide with saturated cotton balls would allow for more precise control of sanitizer application. In addition, while a single treatment with $3 \%$ hydrogen peroxide did appear to offer some reductions in skin ATP biofluorescence, three treatments spaced five minutes apart appeared to work very well to reduce ATP biofluorescence to statistically significant levels. The advantage of $3 \%$ hydrogen peroxide is that it would not as seriously disrupt the skin's lipid bilayer as 70\% aqueous 2-propanol and would minimize the migration of somatic ATP present in the lower layers of the stratum corneum to the skin's surface. It is, however, impossible to differentiate somatic APT from microbial ATP with this testing technique, as both molecules are essentially chemically identical.

It has been shown recently in very interesting work done by Hillebrand et al. that the skin microbiome is very stable over the course of the two years of the study [19]. The work mentioned above by Two also indicates that the skin microbiome is relatively resistant to surfactants [17]. In a recently published study by Peng et al., from the University of Maryland's Department of Animal and Avarian Science, it was demonstrated that while the skin's microbiome can be stable, it can also be influenced significantly by a person's environmental exposures [20]. Peng's work investigated the relationship of the microbiome of farm workers' inner volar forearms to the microbial diversity of various farms in which they worked. It was found that microbial populations can change depending on the individual's environment. In addition, Li et 
al. discussed the impact that aging of the skin has on changes in the microbial diversity of the skin [21]. In a particularly nice summary of the role of the skin's microbiome on skin health, Fourniere et al. discussed the important role of the commensal microorganisms Staphylococcus epidermidis and Cutibacterium acnes in skin health and aging [5]. In an interesting study that appeared recently, Kim et al. examined the influence of various commensal skin microorganisms isolated from the skin of younger people and older people and noted that Streptococcal species were principally more abundant from the skin of the younger test group [22]. They noted that secretions from various streptococcal species stimulated the expression of several genes related to skin barrier function and structure. They related these findings to the expression of spermidine from the bacteria that they felt was responsible for the improvements they noted in the skin.

Recently, a book publication has appeared that delves into the science surrounding the skin microbiome and how it impacts the development of therapeutic and cosmetics products [23]. All this recent work suggests that understanding the role of the skin's microbiome on skin aging and health remains an area of considerable interest and research. However, this has not stopped companies offering cosmetic ingredients from suggesting that topical treatment may balance the skin microbiome or improve the growth of beneficial commensal microorganisms by application of pre- or postbiotic ingredients. The marketing claims for ingredients appear to precede the science around what the skin's microbiome is doing and why it is important in aging and skin disease. It is not uncommon to see cosmetic formulations touting probiotics when the formulations contain microbial or fungal lysates that are not living microorganisms and so are not, technically, probiotics [24].

\section{Conclusion}

With the work presented here, the principal idea was to demonstrate in a small pilot study that ATP biofluorescence might work as a method to rapidly examine skin that has had a microbial disruption imparted to it via a mild sanitizing treatment and to investigate how quickly the normal microbiome might respond to such a dysbiotic treatment. It may be that one of the major impacts of the skin's microbiome and a unique measure of the skin's health is how quickly the skin's surface microbiome can return to normal functionality. Data presented from these initial studies suggest that ATP biofluorescence can offer a way to investigate the global skin microbiome without attention to specific microorganism populations. The results presented here suggest that after a topical disruption, the skin's microbiome may be able to return to a semblance of normality in as little as six hours. Importantly, as the data in Figure 6 demonstrate, within 24 hours of application of the sanitizing treatment, the skin completely returned to the original baseline measurements, indicating that, regardless of whether there may be subtle somatic ATP influences, the skin microbiome returned to pretreatment levels. This suggests that the skin's microbiome is a robust and aggressively adapting system that does not remain disrupted for long in healthy skin. This does raise the question of how this rapid return to normal might become dysfunctional in abnormal skin. 
From a more pragmatic cosmetic and therapeutic perspective, attention to topical treatments that can help to accelerate this microbial repopulation might be a unique area of interest in developing novel topical treatments that can influence the skin's microbiome and health. In addition, the technique may offer a superior way to examine in vivo the concepts around "microbiome-safe", which are currently becoming popular cosmetic claims. As noted, for example, in the work by Gallez et al., the use of ATP bioluminescence to examine gingival microbial populations suggests that it may have applications in numerous areas of research [13]. The mildness of the hydrogen peroxide sanitizing treatments will likely also allow for testing to occur around the face or other sensitive skin areas where the composition of the microbiome is quite different compared to the relatively oil-free surface of the arms.

\section{Abbreviations}

Adenosine Triphosphate (ATP), Ribonucleic Acid (RNA), Hydrogen Peroxide $\left(\mathrm{H}_{2} \mathrm{O}_{2}\right)$, Colony Forming Units (CFU), Relative Fluorescence Units (RFU)

\section{Declarations}

Acknowledgements: N/A

Conflict of Interest: The authors report no conflicts of interest.

Author's Contributions: Both authors contributed equally to the development of the ideas including the testing protocols, analysis of data and writing and editing of the manuscript.

Funding: All funding for the studies were provided by BotanicalsPlus and Jeen International. No additional funding sources are noted.

Availability of Supplemental Materials: N/A

Ethics approval and consent to participate: All volunteers who participated in the studies signed Informed Consent to participate in the studies. All volunteers completed the study, and no volunteers withdrew prematurely from the study protocols.

Consent for publication: Not Applicable

Competing interests: The authors report no competing interests

Author details: JVG is an employee of BotanicalsPlus, Fairfield, New Jersey. JR is an employee of Jeen International, Fairfield, New Jersey.

\section{References}


1. Grice EA, Kong HH, Renaud G, Young AC, NISC Comparative Sequencing Program, Bouffard GG, Blakesley RW, Wolfsberg TG, Turner ML, Segre JA. A diversity profile of the human skin microbiota. Genome Res. 2008;18:1043-50.

2. Flowers L, Grice EA. The Skin Microbiota: Balancing Risk and Reward. Cell Host Microbe. 2020;28:190-200.

3. Myles IA, Earland NJ, Anderson ED, Moore IN, Kieh MD, Williams KW, Saleem A, Fontecilla NM, Welch PA, Darnell DA, Barnhart LA, Sun AA, Uzel G, Datta SK. First-in-human transplantation with Roseomonas mucosa for atopic dermatitis. JCI Insight. 2018;3:e120608.

4. Wang Y, Kuo S, Shu M, Yu J, Huang S, Dai A, Two A, Gallo RL, Huang CM. Staphylococcus epidermidis in the human skin microbiome mediates fermentation to inhibit the growth of Propionibacterium acnes: Implications of probiotics in acne vulgaris. Appl. Microbial Cell Physiol. 2014;98:411-424.

5. Fourniere M, Latire T, Souak D, Feuilloley MGJ, Bedous G. Staphylococcus epidermidis and Cutibacterium acnes: Two major sentinels of skin microbiota and the influence of cosmetics. Microorganisms.2020;8:1752.

6. Leong C, Wang J, Toi MJ, Lam YI, Goh JP, Lee SM, Dawson TL. Effect of zinc pyrithione shampoo treatment on skin commensal Malassesia. Med Mycol. 2021;59:201-213.

7. Dawson TL. Malassezia globosa and restricta: Breakthrough Understanding of the Etiology and Treatment of Dandruff and Seborrheic Dermatitis through Whole-Genome Analysis. J. Invest. Dermatol. 2007;12:15-19.

8. Grogan MD, Bartow-McKenney C, Flowers L, Knight SAB, Uberoi A, Grice EA. Research techniques made simple: Profiling the skin microbiota. J. Invest. Dermatol. 2019;139:747-752.

9. Ferretti P, Farina S, Cristofolini M, Girolomoni G, Tett A, Segata N. Experimental metagenomics and ribosomal profiling of the human skin microbiome. Exp. Dermatol. 2017;26:211-219.

10. Biermann NM, McClure JT, Sanchez J, Saab M, Doyle AJ. Prospective, randomized clinical trial of four different presurgical hand antiseptic techniques in equine surgery. Equine Vet. 2019;51:600-605.

11. Omidbakhsh N, Ahmadpour F, Kenny N. How reliable are ATP bioluminescence meters in assessing decontamination of environmental surfaces in healthcare settings? PLoS One. 2014;9:e99951.

12. Ihssen J, Jovanovic N, Sirec T, Spitz U. Real-time monitoring of extracellular ATP in bacterial cultures using thermostable luciferase. PLoS One. 2021;16:e0244200.

13. Gallez F, Fadel M, Scruel O, Cantraine F, Courtois P. Salivary biomass assessed by bioluminescence ATP assay related to (bacterial and somatic) cell counts. Cell Biochem. Funct. 2000;18:103-108.

14. Johnson A, Papnicki P, Farnsworth R, Stewart S, Salter R, Sigurdson C, Adkins L, Burnett S. Measuring effectiveness of teat preparation. Univ. Minn. Coll. Vet. Med. Minn. Dairy Heath Conf. (2003) https://conservancy.umn.edu/bitstream/handle/11299/108987/1/Johnson.pdf.

15. Siragusa GR, Dorsa WJ, Cutter CN, Perino LJ, Koohmaraie M. Use of a newly developed rapid microbial ATP bioluminescence assay to detect microbial contamination on poultry carcasses. J. Biolumin. Chemilumin. 1996;11:297-301. 
16. Kwan SE, Peccia J, Simonds J, Haverinen-Shaughnessy U, Shaughnessy RJ. Comparing bacterial, fungal, and human cell concentrations with rapid adenosine triphosphate measurements for indicating microbial surface contamination. Am. J. Infect. Cont. 2019;47:671-676.

17. Two AM, Nakatsuji T, Kotol PF, Arvanitidou E, Du-Thumm L, Hata TR, Gallo RL. The cutaneous microbiome and aspects of skin antimicrobial defense system resists acute treatment with topical cleansers. J. Invest. Dermatol. 2016;136:1950-1954.

18. Liceaga A, Mercado E, Narcaroti M, Liceaga L. Hand hygiene skin ATP study: Hand washing with tap water and soap vs ozonated water and soap vs antiseptic and tap water vs antiseptic and ozonated water vs antiseptic and tap water. Becker's Hospital Review (2017).

https://www.beckershospitalreview.com/quality/hand-hygiene-skin-atp-study-hand-washing-with-tapwater-and-soap-vs-ozonated-water-and-soap-vs-antiseptic-and-ozonated-water-vs-antiseptic-and-tapwater.html.

19. Hillebrand GG, Dimitriu PD, Malik K, Park Y, Qu D, Mohn WW, Kong R. Temporal variation of the facial skin microbiome: A 2-year longitudinal study in healthy adults. Plast. Reconstr. Surg. 2021;147:50S.

20. Peng M, Biswas D. Environmental influences of high-density agricultural animal operation on human forearm skin microflora. Microorganisms. 2020;8:1481.

21. Li Z, Bai X, Peng T, Yi X, Lou L, Yang J, Liu J, Wang Y, He T, Wang X, Zhu H, Wang H, Tao K, Zheng Z, Su L, Hu D. New insights into skin microbial communities and skin aging. Front Microbiol. 2020;11:565549.

22. Kim G, Kim M, Kim M, Park C, Yoon Y, Lim D-H, Yeo H, Kang S, Lee Y-G, Lee BJ, Kim S, Kwon JY, Choi WW, Lee C, Yoon KW, Park H, Lee D-G. Spermidine-induced recovery of human dermal structure and barrier function by skin microbiome. Commun. Biol. 2021;4:231.

23. Skin Microbiome Handbook: From Basic Research to Product Development. Nava Dayan, Ed., John Wiley \& Sons, Pub., Hoboken, NJ (2020).

24. Yu Y, Dunaway S, Champer J, Kim J, Alikhan A. Changing our microbiome: probiotics in dermatology. Brit J Dermatol. 2020;182:39-46.

\section{Figures}


ATP Biofluorescence Relative Fluorescent Units (RFU) Averages, N=30 Arms, $p<0.05$

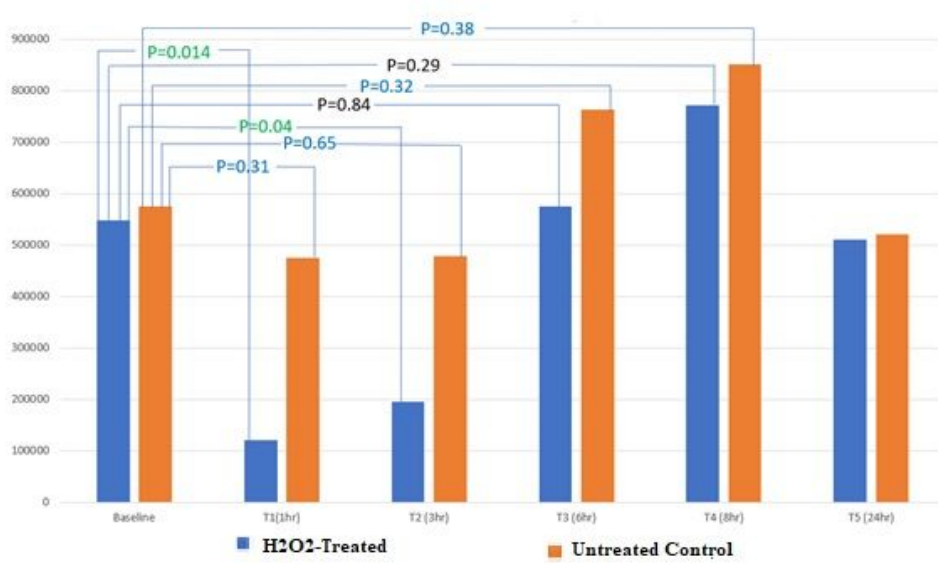

Figure 1

Raw ATP Bioluminescence Relative Fluorescent Unit Averages for each arm site and statistical analyses comparing the Baseline measurements versus hydrogen peroxide-treated sites at each time point and the H2O2-treated versus Untreated Control sites at each time point except the 24-hour time point, which is discussed in greater detail in Figure 3. Only the H2O2-treated baseline versus 1-hour and 3-hour sites (green p-values) showed statistically significant differences, indicating that the $3 \%$ hydrogen peroxide treatment reduced the overall level of ATP bioluminescence at these sites versus the untreated control sites. $p<0.05$ 
Baseline normalized ATP Biofluorescence Results, N=30 Arms, $p<0.05$

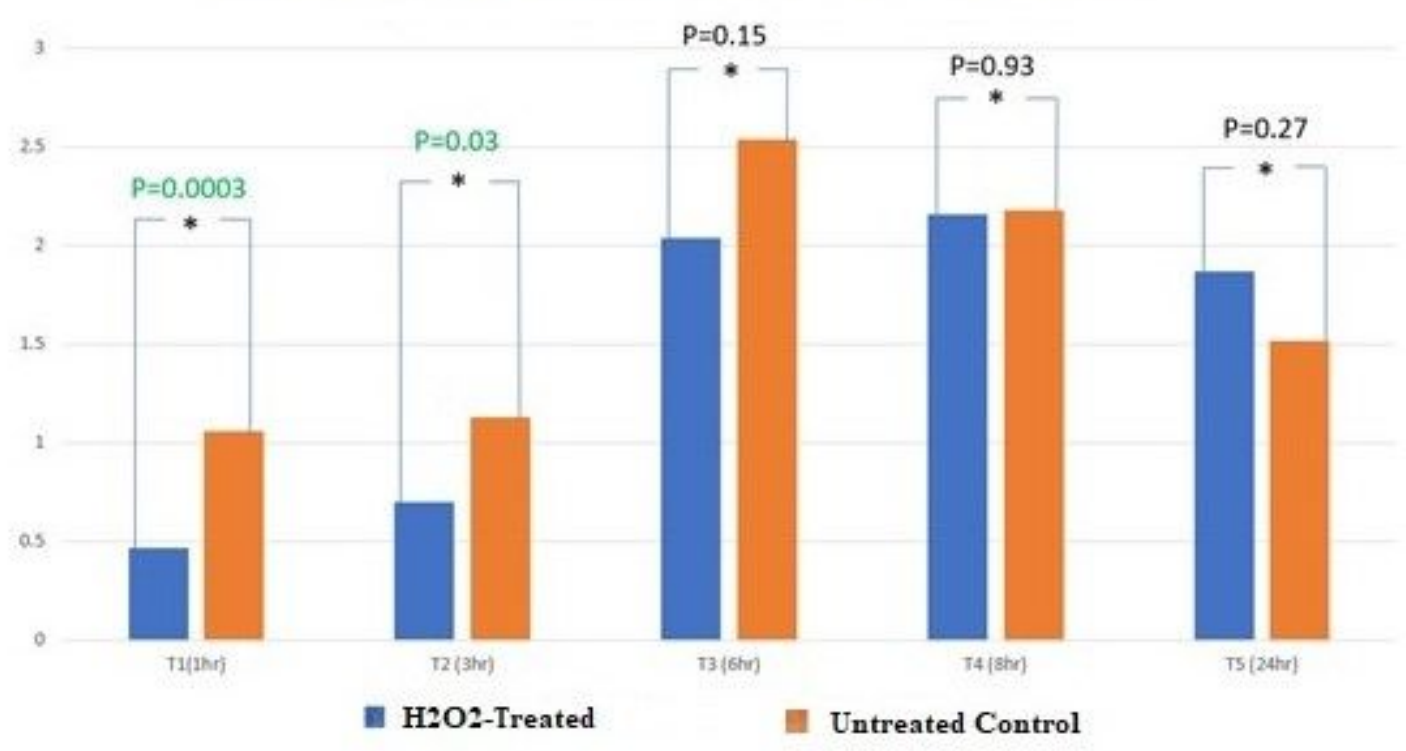

Figure 2

Results of ATP fluorescent (RFU) measurements normalized to Baseline for timepoints $T(1), T(2), T(3)$, $\mathrm{T}(4)$ and $\mathrm{T}(5)$ showing statistical results comparing H2O2-treated sites (blue) versus untreated control sites (orange). $\mathrm{N}=30, \mathrm{p} \leq 0.05$.

ATP Biolumenescence RFU: Baseline vs $24 \mathrm{Hr}$

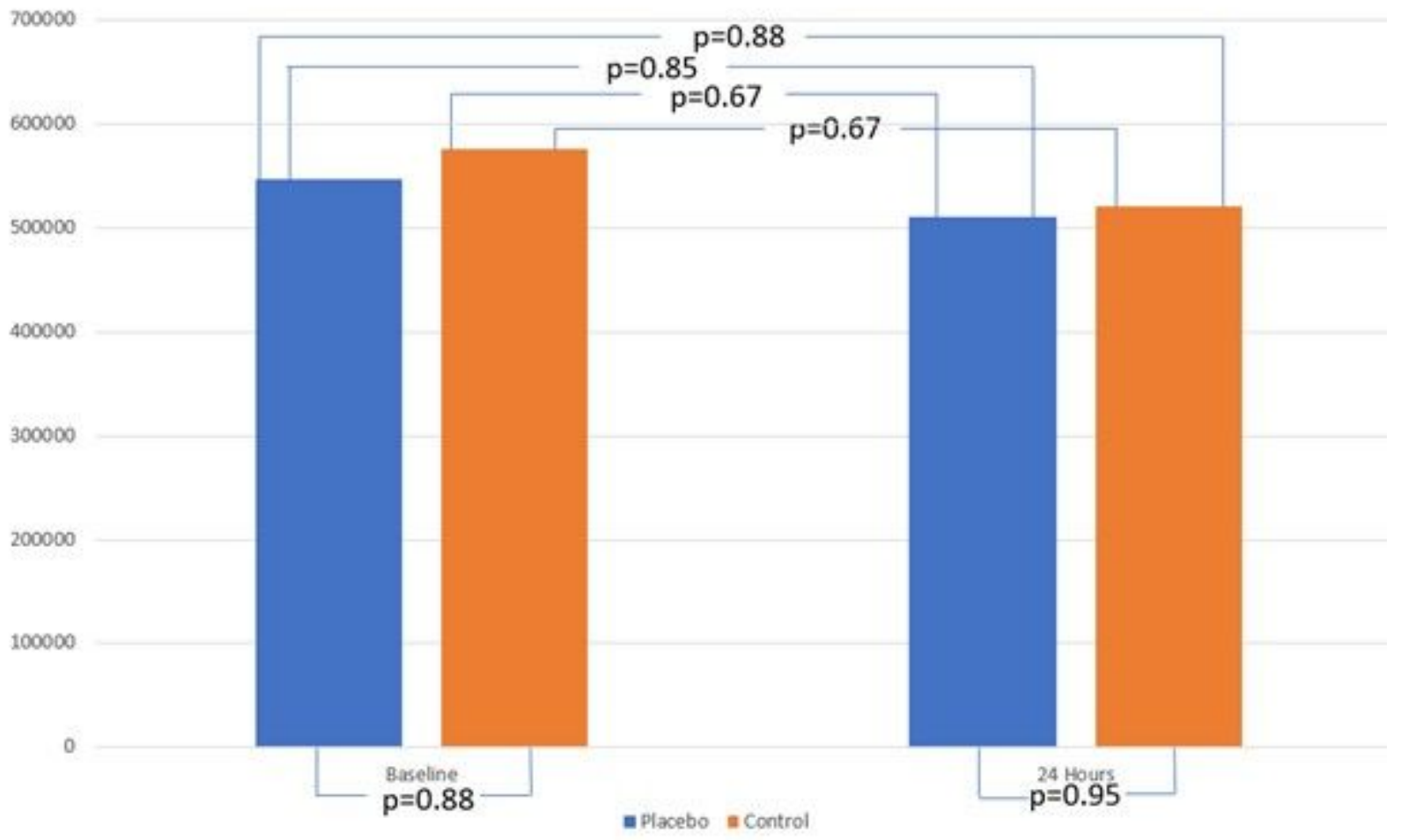

Figure 3 
Relative Fluorescence Unit (RFU) results comparing initial baseline measurements against similar measurements 24 hours after $3 \%$ hydrogen peroxide dysbiosis. $N=30, p \leq 0.05$.

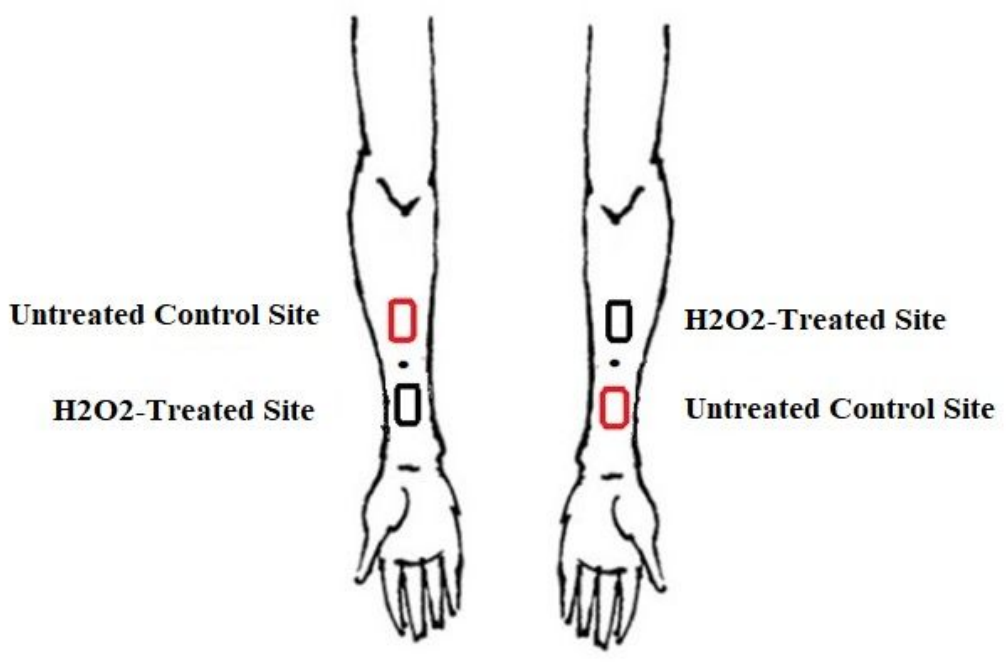

Figure 4

Schematic diagram showing how both arms on the volunteers were marked and the locations where $3 \%$ hydrogen peroxide-treated sites were alternately applied and where the untreated control sites were left untreated.

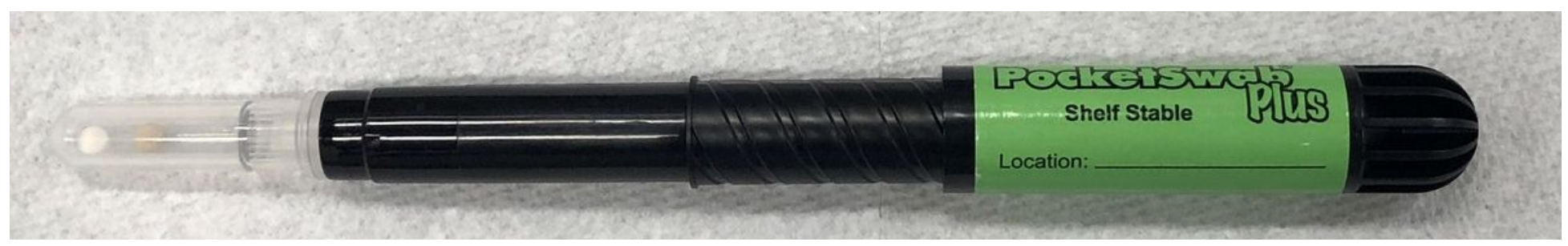

Figure 5

Charm Sciences Pocketswab Plus unit. 


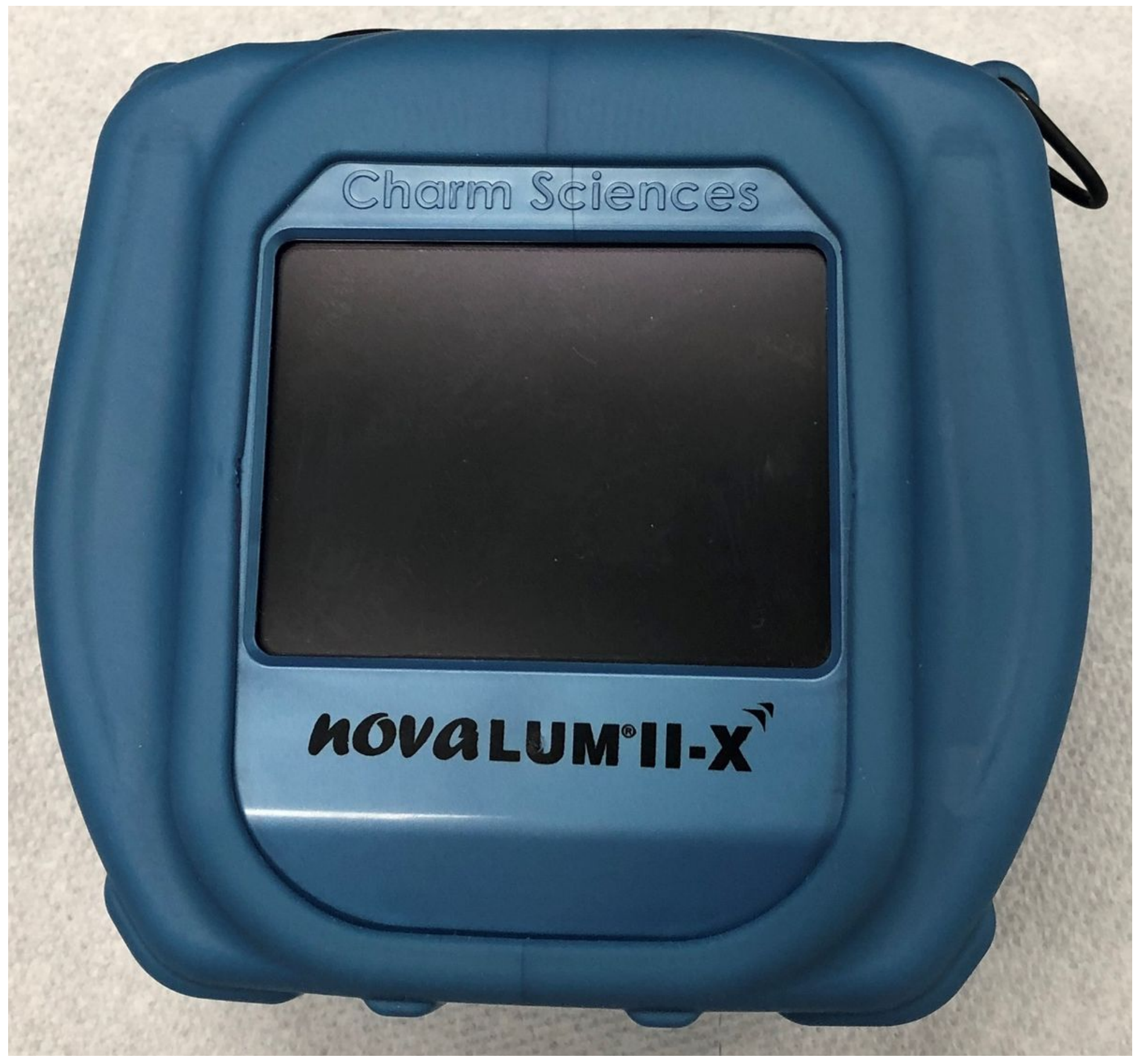

Figure 6

Charm Novalum II-X ATP Biofluorescence Measuring Unit 